\title{
Building Economic Models and Measures of Search
}

\author{
Leif Azzopardi \\ University of Strathclyde \\ Glasgow, UK \\ leifos@acm.org \\ Paul Thomas \\ Microsoft \\ Canberra, Australia \\ pathom@microsoft.com
}

\author{
Alistair Moffat \\ The University of Melbourne \\ Melbourne, Australia \\ ammoffat@unimelb.edu.au \\ Guido Zuccon \\ The University of Queensland \\ Brisbane, Australia \\ g.zuccon@uq.edu.au
}

\begin{abstract}
Economics provides an intuitive and natural way to formally represent the costs and benefits of interacting with applications, interfaces and devices. By using economic models it is possible to reason about interaction, make predictions about how changes to the system will affect behavior, and measure the performance of people's interactions with the system. In this tutorial, we first provide an overview of relevant economic theories, before showing how they can be applied to formulate different ranking principles to provide the optimal ranking to users. This is followed by a session showing how economics can be used to model how people interact with search systems, and how to use these models to generate hypotheses about user behavior. The third session focuses on how economics has been used to underpin the measurement of information retrieval systems and applications using the $\mathrm{C} / \mathrm{W} / \mathrm{L}$ framework (which reports the expected utility, expected total utility, expected total cost, and so on) - and how different models of user interaction lead to different metrics. We then show how information foraging theory can be used to measure the performance of an information retrieval system - connecting the theory of how people search with how we measure it. The final session of the day will be spent building economic models and measures of search. Here sample problems will be provided to challenge participants, or participants can bring their own.
\end{abstract}

\section{ACM Reference Format:}

Leif Azzopardi, Alistair Moffat, Paul Thomas, and Guido Zuccon. 2019. Building Economic Models and Measures of Search. In Proceedings of the 42nd International ACM SIGIR Conference on Research and Development in Information Retrieval (SIGIR '19), July 21-25, 2019, Paris, France. ACM, New York, NY, USA, 2 pages. https://doi.org/10.1145/3331184.3331379

\section{MOTIVATION}

Economics provides an intuitive and natural way to formally represent the costs and benefits of interaction. Over the years, various economic concepts have been used with Information Retrieval (IR) to provide: (i) the underpinnings of retrieval models (e.g., the probability ranking principle [19], etc.); (ii) the development of new retrieval models (e.g., portfolio theory [23], facilities location analysis [27], etc.); (iii) the measurement of ranked lists (e.g., discounted cumulative gain [11], expected utility [12], etc.); and (iv) the modelling of user behaviors (e.g., information foraging theory [17], production theory [1], etc.). By exploring how economic theory has permeated the field of IR, the goal of this tutorial is to provide the audience with an overview of the key developments, showing how the underlying economics concepts can be applied to: (1) build models of search behavior; and (2) develop metrics to search performance. By using economic models it is possible to reason about interaction, make predictions about how changes to the system affect behavior, and measure the performance of people's interactions with the system.

\section{OBJECTIVES}

From this tutorial a student should learn how to:

- Describe how economics has influenced developments in IR;

- Compare and contrast the different ranking principles;

- Describe different models of user behavior;

- Create an economic model of search;

- Explain the $\mathrm{C} / \mathrm{W} / \mathrm{L}$ framework and the different measurements it incorporates;

- Design a metric given the $\mathrm{C} / \mathrm{W} / \mathrm{L}$ framework; and

- Infer, hypothesize and predict user behaviors and performance.

\section{COURSE FORMAT}

The full day tutorial at SIGIR 2019 consists of four sessions.

\subsection{Session 1 - Economics in IR}

The first session focuses on providing a grounding in economics and optimization models $[10,16]$. It explains why we need such models, and how they can be used to gain insights about search performance and search behavior. We then provide a high level overview of how different economics concepts and theories have been utilized in IR, in terms of: (1) ranking documents (e.g., ranking principles); (2) modelling user behavior (e.g., user models); and (3) measuring performance (e.g., metrics and measurements).

The remainder of the session takes a deeper dive into the economics behind ranking principles $[9,19,25,26]$. Starting from the Probability Ranking Principle (PRP), we show how this decisiontheoretic approach ensures that documents are ranked optimally 
(given a number of assumptions). We then explain how the PRP's assumptions about user behavior can be relaxed and how it can be extended to be more realistic, by detailing the Interactive Probability Ranking Principle [9] and the Generalized PRP, that is, the Card Model [24, 25]. In summary, this session provides the foundations for understanding how economics has guided and underpinned the development of ranking models.

\subsection{Session 2 - Economic Models of Search}

In session two, we change focus, and examine models that are userfocused. That is, we provide an overview of economic models that aim to explain and predict user search behavior [1-3, 17]. To this end, we describe Information Foraging Theory [17, 18, 20, 21] (IFT), which applies economics to how people find and forage for information (based on Optimal Foraging Theory [22] from ecology). We explain how IFT can more generally be used to model how people search and forage for information by trying to maximize their rate of gain over time (e.g., Charnov's Marginal Value Theorem [7]) While IFT has been used to motivate experimentation at the high level, we explain more concretely how IFT can be used to derive testable hypotheses about how people interact with an information system, and how their behavior adapts depending on the circumstances. We then continue working through different economic models that have been developed to represent different interfaces, features and scenarios. This session puts the theory into practice showing how different costs and benefits impact usage, and performance in a number of contexts $[2-4,8]$ ). By using economic models it is possible to draw actionable insights regarding how to improve the system and to identify and understand the trade-offs between interactions (to explain when and why one option is preferred or used over another).

\subsection{Session 3 - Economic Measures of Search}

In the third session, we examine how economics can be used to evaluate the user-system interactions. Most existing metrics essentially report the expected utility (also called the rate of gain) given the ranked list and model of user behavior. Here we link the models of search behavior with how we measure search performance by describing and explaining the $\mathrm{C} / \mathrm{W} / \mathrm{L}$ framework, which utilizes economics theory to provide the theoretical basis for measuring Expected Utility [13, 15], as well as a series of other related economic measures [5]. We then take a deeper dive into the $\mathrm{C} / \mathrm{W} / \mathrm{L}$ framework, and explain how different metrics, encode different user models (e.g., P@k, DCG, RBP, and INST [5, 13-15]) and as such how to design and build your own metric [6]. Finally, we conclude the session by showing how models of search behavior (i.e., IFT) can be used to define theoretically underpinned metrics within the C/W/L framework - connecting "how we model search" with "how we measure search".

\subsection{Session 4 - Practical Session}

The final session of the tutorial is dedicated to a hands-on practical session where we challenge participants to apply economics modelling techniques to different scenarios - where they will need to model user behavior in order to hypothesize in regard to how people interact with the system, or to evaluate how well people perform using the system.

\section{REFERENCES}

[1] Leif Azzopardi. The economics in interactive information retrieval. In Proc. of the 34th International ACM SIGIR Conference, pages 15-24, 2011.

[2] Leif Azzopardi. Modelling interaction with economic models of search. In Proc. of the 37th International ACM SIGIR Conference, pages 3-12, 2014.

[3] Leif Azzopardi and Guido Zuccon. An analysis of the cost and benefit of search interactions. In Proc. of the 2016 ACM International Conference on the Theory of Information Retrieval, ICTIR '16, pages 59-68, 2016.

[4] Leif Azzopardi and Guido Zuccon. Two scrolls or one click: A cost model for browsing search results. In Proc. of the 38th European Conference on IR Research, pages 696-702, 2016.

[5] Leif Azzopardi, Paul Thomas, and Nick Craswell. Measuring the utility of search engine result pages: An information foraging based measure. In Proc. of the 41st International ACM SIGIR Conference, SIGIR '18, pages 605-614, 2018.

[6] Leif Azzopardi, Paul Thomas, and Alistair Moffat. cwl_eval: An evaluation tool for information retrieval. In Proc. of the 42nd International ACM SIGIR Conference, SIGIR '19, 2019.

[7] Eric L. Charnov. Optimal foraging, the marginal value theorem. Theoretical Population Biology, 9(2):129-136, 1976.

[8] Michael D. Cooper. A cost model for evaluating information retrieval systems. Journal of the American Society for Information Science, pages 306-312, 1972.

[9] Norbert Fuhr. A probability ranking principle for interactive information retrieval. Information Retrieval, 11(3):251-265, 2008.

[10] Frederick S. Hillier and Gerald J. Lieberman. Introduction to Operations Research. McGraw-Hill, NY, USA, 2001.

[11] Kalervo Järvelin and Jaana Kekäläinen. Cumulated gain-based evaluation of IR techniques. ACM Trans. Inf. Syst., 20(4):422-446, October 2002.

[12] Alistair Moffat and Justin Zobel. Rank-biased precision for measurement of retrieval effectiveness. ACM Trans. Inf. Syst., 27(1):2:1-2:27, 2008.

[13] Alistair Moffat, Paul Thomas, and Falk Scholer. Users versus models: What observation tells us about effectiveness metrics. In Proc. of the ACM International Conference on Information and Knowledge Management, pages 659-668, 2013.

[14] Alistair Moffat, Peter Bailey, Falk Scholer, and Paul Thomas. INST: An adaptive metric for information retrieval evaluation. In Proc. of Australasian Document Computing Symposium, pages 5:1-5:4, 2015.

[15] Alistair Moffat, Peter Bailey, Falk Scholer, and Paul Thomas. Incorporating user expectations and behavior into the measurement of search effectiveness. ACM Trans. Inf. Syst., 35(3):24:1-24:38, 2017.

[16] Katta G. Murty. Optimization Models For Decision Making. University of Michigan, Ann Arbor, 2003.

[17] Peter Pirolli and Stuart Card. Information foraging. Psychological Review, 106: 643-675, 1999.

[18] Howard L. Resnikoff. The Illusion of Reality. Springer-Verlag New York, 1989.

[19] Stephen E. Robertson. The probability ranking principle in IR. Journal of Documentation, 33(4):294-304, 1977.

[20] Daniel M. Russell, Mark J. Stefik, Peter Pirolli, and Stuart K. Card. The cost structure of sensemaking. In Proc. of the INTERACT and SIGCHI, pages 269-276, 1993.

[21] Pamela Effrein Sandstrom. An optimal foraging approach to information seeking and use. The Library Quarterly, pages 414-449, 1994.

[22] D. W. Stephens and J. R. Krebs. Foraging Theory. Princeton University Press, 1986.

[23] Jun Wang and Jianhan Zhu. Portfolio theory of information retrieval. In Proc. of the 32nd International ACM SIGIR Conference, pages 115-122. ACM, 2009.

[24] ChengXiang Zhai. Towards a game-theoretic framework for information retrieval. In Proc. of the 38th International ACM SIGIR Conference, pages 543-543, 2015

[25] Yinan Zhang and ChengXiang Zhai. Information retrieval as card playing: A formal model for optimizing interactive retrieval interface. In Proc. of the 38th International ACM SIGIR Conference, SIGIR '15, pages 685-694, 2015.

[26] Guido Zuccon, Leif A Azzopardi, and Keith Rijsbergen. The quantum probability ranking principle for information retrieval. In Proc. of ICTIR, pages 232-240, 2009.

[27] Guido Zuccon, Leif Azzopardi, Dell Zhang, and Jun Wang. Top-k retrieval using facility location analysis. In Proc. of the 34th European Conference on IR Research, pages 305-316. 2012. 